\title{
Virtual Atomic and Molecular Data Centre: Level 3 Service and Future Prospects
}

\author{
M. L. Dubernet ${ }^{1}$, G. Rixon ${ }^{2}$, M. Doronin ${ }^{1}$ and VAMDC \\ Collaboration $\dagger$ \\ ${ }^{1}$ LPMAA, UMR7092, Université Pierre et Marie Curie, and Observatory of Paris, France \\ email: marie-lise.dubernet@obspm.fr \\ ${ }^{2}$ Institute of Astronomy, University of Cambridge, Madingley Road, Cambridge, CB30HA, UK
}

\begin{abstract}
The Virtual Atomic and Molecular Data Centre (VAMDC, http://www.vamdc.eu) is an international Consortium that has created an interoperable e-science infrastructure for the exchange of atomic and molecular data. The VAMDC defines standards for the exchange of atomic and molecular data, develop reference implementation of those standards, deploys registries of internet resources (yellow pages), designs user applications in order to meet the user needs, builds data access layers above databases to provide unified outputs from these databases, cares about asynchronous queries with workflows and connects its infrastructure to the grid. The paper describes the current service deployment of the VAMDC data infrastructure across our registered databases and the key features of the current infrastructure.
\end{abstract}

Keywords. atomic data, molecular data, standards, data bases

\section{Level 3 Release of VAMDC, September 2012}

A level-n release is a combined release of: standards for data access, the VAMDC nodes, each containing a database and web service following the standards, a registry of the services, a web portal as UI for the system, softwares to prepare the databases (Node Software) and implement the web services, web services to process VAMDC results into other formats and presentations (Consumer Service), update of VAMDC tools. Rixon et al. (2011) and Doronin et al. (2012) describe Level 1 and Level 2 services respectively, thus providing the definition of the above concepts, while Dubernet et al. (2010) is the funding paper describing the VAMDC consortium.

The level-3 release contains the resources made available to users in VAMDC on September 2012, based on the 2011.12 release of standards. The standards for the 2011.12 release (http://vamdc.eu/standards) specify: the data model and format XSAMS, in the variant currently supported by VAMDC, called VAMDC-XSAMS version 0.3 , the webservice protocol VAMDC-TAP, the query language VSS2, the VAMDC dictionary of standard terms, the method of registering nodes, and the protocol for XSAMS-consuming services. The level 3 databases are registered in the level 3 registry (http://registry.vamdc.eu/registry-11.12/) which is separate from the registries for other releases and from the registry used for development of the system. The VAMDC portal (http://portal.vamdc.eu) always reads the latest released registry.

\section{What can you do with VAMDC services}

- Use our graphical User Interface, called portal (http://portal.vamdc.eu) to query all currently registered VAMDC databases, and download or visualize the data. The portal

$\dagger$ See References Dubernet et al. (2010), Rixon et al. (2011), Doronin et al. (2012) 
usage is supported by different e-tutorials that can be found at http://vamdc.eu/usersupport. It should be noted that the "consumer services" which transform the VAMDC-XSAMS files into other formats are independent of the portal and users may offer to connect their own consumer service to the portal.

- Implement your own database within VAMDC using the Node Software in Python or Java (Doronin et al, 2012)

- Implement our librairies and/or standards in your own application software : you may use the on-line documentation, download the different libraries and software.

- Use our currently available tools (http://vamdc.eu/software): TAPValidator to check the output of VAMDC nodes, SPECTCOL to combine collisional data with spectrocopic data for the study of non-LTE interstellar media.

- Get support for all these features and propose new features or tools

\section{Support for Users}

The section User Support (http://vamdc.eu/usersupport) provides the access to the support mailing list, and to training materials currently including the use of Portal, the use of Taverna workflow, the use of the GRID. The documentation for standards are found at http://vamdc.eu/standards, the documentation related to software are included with the software (http://vamdc.eu/software). For any kind of request, bug report, question on the operation, support to user, or general enquiries, please e-mail support@vamdc.eu. Your email will be placed as a ticket to be looked at by the VAMDC support-community and an answer will come back as soon as possible.

\section{Future Prospect}

At the time of writing this paper the standards version 2012.07 have been released, the node software in Java version 12.07r1, the TAPValidator Tool v12.07, the client SPECTCOL version 12.07 have been released, and Level-4 release, based on standards v12.07, is planned for mid-december 2012. From December 2012 the VAMDC consortium will provide new n-level releases every year at most, so that stability of software is garantied.The VAMDC is further suppported for 2 years by a new european grant "SUP@VAMDC" (http://sup.vamdc.eu) that will allow to support the already existing infrastructure and to extend its usage by new producers of data in a wider range of countries, as well as by new users including high education, schools, citizens and SMEs.

\section{Acknowledgements}

VAMDC and SUP@VAMDC are funded under the Combination of Collaborative Projects and Coordination and Support Actions Funding Scheme of The Seventh Framework Program. Call topic: INFRA-2008-1.2.2 and INFRA-2012 Scientific Data Infrastructure. Grant Agreement numbers: 239108 and 313284.

\section{References}

Dubernet, M. L., Boudon, V., Culhane, J. L., et al. 2010, JQSRT 111, 2151

Doronin, M. \& Dubernet, M. L., al 2012, in ASP Conf. Ser., ADASS XXI, ed. P. Ballester, D. Egret, and N. P. F. Lorente (San Francisco: ASP) 461, p.331

Rixon, G., Dubernet, M. L., Piskunov, N., et al. 2011, in American Institute of Physics Conference Series, American Institute of Physics Conference Series, ed. A. Bernotas, R. Karazija, E3 Z. Rudzikas, 1344, p.107-115 